\title{
Collective motion of bacteria and their dynamic assembly behavior
}

\author{
Jingjing Feng and Yan $\mathrm{He}^{*}$
}

\begin{abstract}
In recent years, active matter systems have attracted considerable attentions due to their complex dynamic behaviors in physical and material science. In particular, microorganism systems have served as model systems for observing dynamic assembly and collective motility of active particles and significant progresses have been made on in-depth understanding of how high density bacteria colony behaves in the non-equilibrium state. In this mini-review, we mainly focus on the collective motion of bacteria and their dynamic assembly from four aspects: (1) the general phenomenon and biological mechanism of bacterial collective motion; (2) the common experimental techniques for studying bacterial motility; (3) some active systems on exploring bacterial collective behavior, which include both non-restricted free suspensions and those in relative confined geometric space; (4) the phenomenological and descriptive statistical methods and physical models on the underlying laws that lead to large-scale coordinate patterns in multicellular systems. This review aims to give a general picture of the collective motion in bacterial active matter systems experimentally and theoretically in order to reflect the interplays between individuals among populations in motion. It is expected that the general regulation rules related to the boundary effects in the complex systems and materials can be elucidated to some extent.
\end{abstract}

Keywords: active matter, bacterial swarming, collective behavior

\section{INTRODUCTION}

Different from most natural or synthetic materials, an active matter system whose structure and function are governed by a static or localized energy landscape, usually refers to a collection or suspension of self-propelled particles capable of acquiring energy either from the external environment or from internal conversion to generate mechanical forces and viscous flow, thus driving the system into a non-equilibrium state. The unique feature of the state is then manifested as movement, growth or reproduction of active particles. In general, active systems containing high concentration of active particles tend to move and assemble coordinately under some asymmetry-breaking mechanism, resulting in organized pattern formations and collective behaviors (Fig. 1) such as large areas of swirls, flocks, plumes, and vortices etc. [1-6]. In addition, there exist other complex-life-like behaviors, e.g. turbulences under non-inertial conditions [7], huge density fluctuations [8] and orientational arrangements like liquid crystal molecules [9].

These dynamic self-organizations due to local interactions with the fluid media have arose strong interests of many researchers. They usually exhibit three characteristics: first, the system contains a handful of components which can contact through non-covalent interactions; second, they should be far from equilibrium; third, there should be abundant energy supply in order to maintain repeated moving patterns.

Traditionally, the active matter system can be divided into many different categories: living cells [10], microtubule system consisting of actin and myosin [11], undomesticated bacterial system [12], self-oscillated polymer gels [13], synthetic Janus particles [14-17] such as $\mathrm{Au}-\mathrm{Pt}$ rods and many other types [18-22] (Fig. 2). As a typical model object with the nature of "self-propelled particle", the bacteria are usually micron-sized and could generate collective motion by taking energy from nutrients in the culture medium.

The study of bacterial motility and collective motion is the necessary prerequisite to learn the spatial and temporal correlation of the complex system and to monitor the assembly of biological systems. Many people have studied

\footnotetext{
Department of Chemistry, Tsinghua University, Beijing 100084, China

${ }^{*}$ Corresponding author (email: yanhe2021@mail.tsinghua.edu.cn)
} 
a

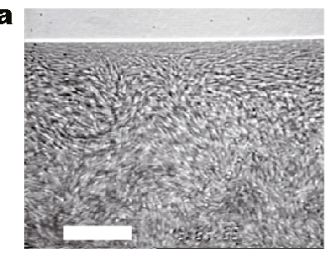

$\mathbf{b}$

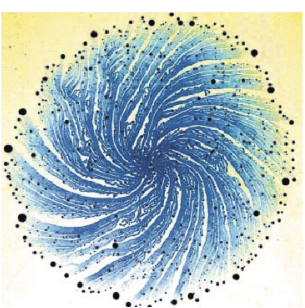

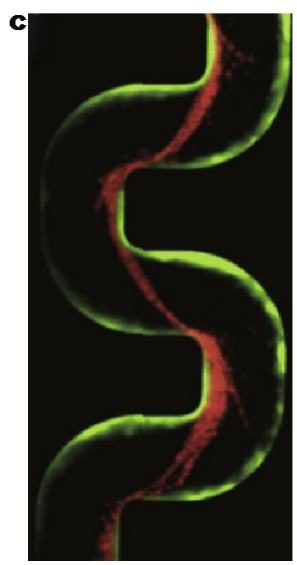
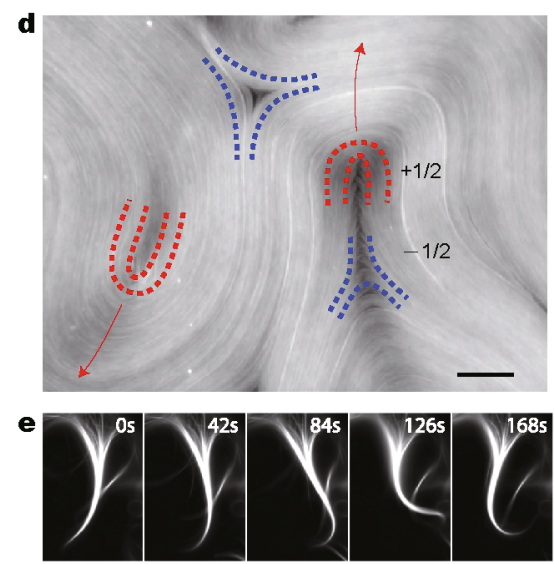

Figure 1 Examples of collective behavior. (a) In water, B. subtilis exhibits collective motion. Scale bar is approximately $15 \mu \mathrm{m}$. Reprinted with permission from Ref. [1]. Copyright 2004, the American Physical Society. (b) Assembled vortex on agar. Reprinted with permission from Ref. [2]. Copyright 2001, Nature Publishing Group. (c) Bacterial biofilm streamers lead to blockage in channels. Reprinted with permission from Ref. [3]. Copyright 2016, Elsevier. (d) The active nematic behavior of the microtubule system with defects. Reprinted with permission from Ref. [4]. Copyright 2015, Nature Publishing Group. (e) Microtubule systems generate metachronal waves. Reprinted with permission from Ref. [5]. Copyright 2011, the American Association for the Advancement of Science.

a

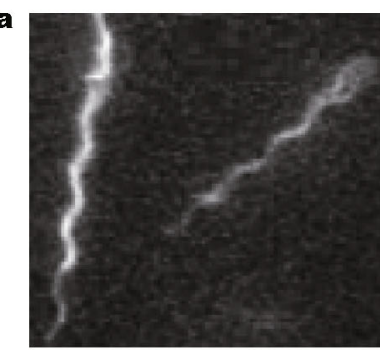

b
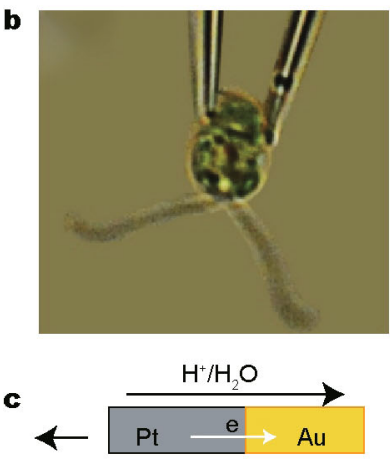

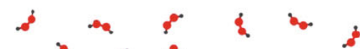

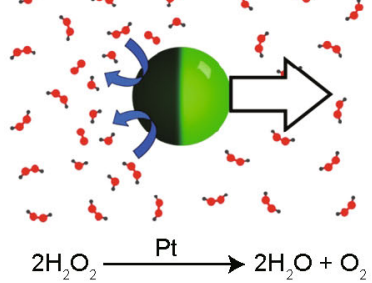

d

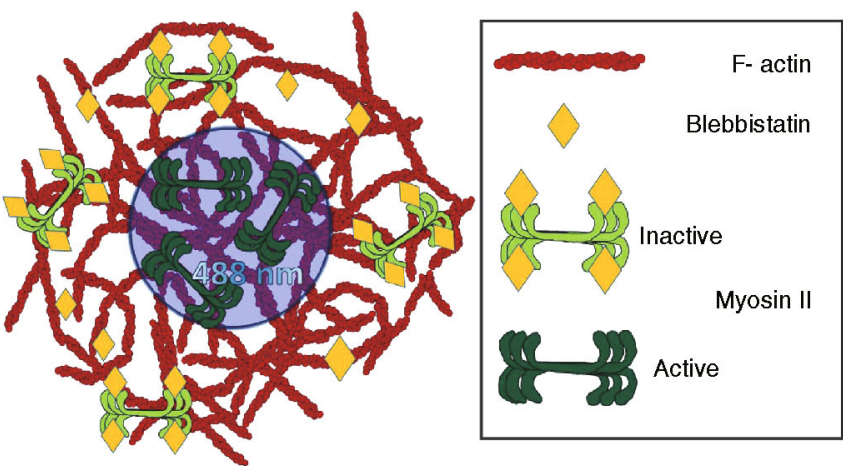

e

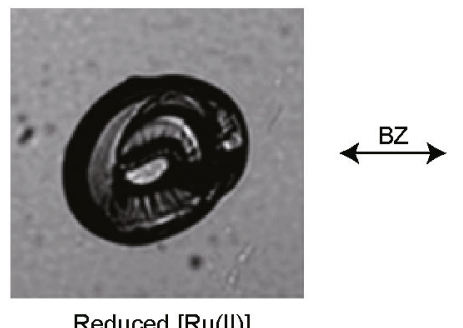

Reduced [Ru(II)]

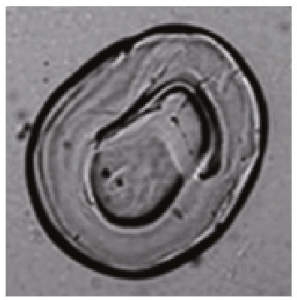

Oxidized [Ru(III)]

f
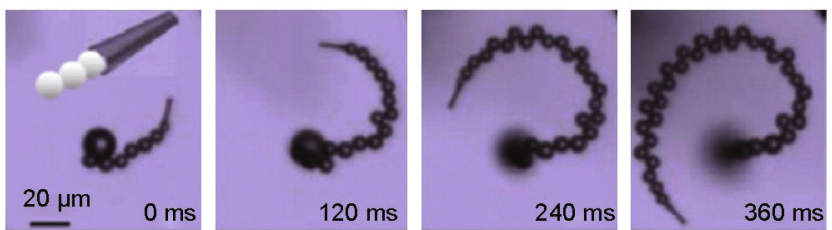

Figure 2 Different kinds of active matter systems. (a, b) Examples of active bacteria. Reprinted with permission from Ref. [3]. Copyright 2016, Elsevier. (a) Escherichia coli with cell body approximately $2 \mu \mathrm{m}$. (b) Chlamydomona s reinhardtii with a cell body that is approximately $8 \mu \mathrm{m}$. (c) Synthetic Au-Pt nanorods and spherical active Janus colloid. Reprinted with permission from Ref. [14]. Copyright 2016, Elsevier. (d) Active actin gels. Reprinted with permission from Ref. [11]. Copyright 2016, Nature Publishing Group. (e) Self-oscillated polymer gels. Reprinted with permission from Ref. [13]. Copyright 2014, the American Chemical Society. (f) Tubular active colloids. Reprinted with permission from Ref. [14]. Copyright 2016, Elsevier. 
the collective motility of bacteria from different levels and aspects, i.e. focusing on the individual behavior among the groups, regarding the motion as a whole and monitoring them in both unrestricted spaces [7] and in micro channels similar to bacteria's living environment [8]. Kearns [23] wrote a comprehensive field guide to bacterial swarming motility. Harshey [24] reviewed the collective movements of bacteria over surfaces including different types, initiation and migration of their surface motility in detail. Copeland et al.[25] emphasized that biological and biophysical mechanisms played an important role in bacterial coordinative movements. Verstraeten et al. [26] focused on the biofilm formation and the transition between motile and sessile lifestyles of bacteria. Koch et al. [27] stressed the role of hydrodynamics in bacterial swarming through concluding theoretical and simulation studies. These investigations have revealed the physical mechanisms of bacterial movement as well as the chemotaxis effects.

\section{THE PHENOMENON AND BIOLOGICAL MECHANISM OF BACTERIAL COLLECTIVE MOTILITY}

The motility of bacteria is closely related to their own biological structure. The flagellum of bacteria plays an important role in its motility. Flagellum mainly consists of three parts: (1) helical filament; (2) hook; (3) basal body and motor components. The basal body, which consists of four rings and one rod, is inserted into the membrane. The motor on the flagellum consists of stators (MotA and MotB) and rotors (FlgB, FlgC, FlgF, and FlgG) (Fig. 3a). The stationary stator is inserted into the basal body and the rotor is connected to the MS-ring [25,28]. In this way, the stator and rotor are combined to generate torque on its body. When the motor rotates counterclockwise, it will cause the left-hand helical flagellum to form a bundle, thus pushing the bacteria forward. When one or more rotors rotate clockwise, a right-hand spiral is formed and then the flagellum bundle is released. At this time, the bacteria itself will adjust its direction of the movement. This kind of movement mechanism of a single bacterium in the fluid is defined as "run and tumble" mechanism (Fig. 3c, d). Saragosti et al. [29] explored the reorientation dynamics and found that the motion of bacteria appeared as random walk on a unit sphere and the tumbles could be described by their rotational diffusion coefficient. The average direction change was 68 degrees and the turning time was about 0.14 s. The bacterial motility is also associated with their various physiological activities such as elongation, increased flagellar density, secretion of surfactants [12] and enhanced antibiotic resistance [30].

On the basis of the biological structure, the collective behavior of bacteria colony usually requires some necessary conditions. To certain extent, the bacteria must have a flagellar structure and a relative high concentration to enable mutual contact.

The behavior of the collective movement (Fig. 3b) of bacteria could occur in different culture systems. For example, one could fabricate a free-standing film after culturing the bacteria in broth. Alternatively, the free rod-shaped bacterial suspensions with high density could be directly added onto solid surfaces such as agar. Indeed, bacteria using flagella to move on agar surfaces are usually referred as "bacterial swarming" by researchers. The bacterial swarming behavior is magnified as vortices, swirls and jets [23] by microscopic techniques. These phenomena can sustain from seconds to hours due to the difference in bacterial species or the difference between the culture system and the environmental conditions.

Overall, this long-range coupled population behavior of bacteria may be related to many factors [31]. The most common aspect is the physical interaction inside the system, including the interaction of bacteria with the surrounding fluid, as well as the collision between bacteria and the steric hindrance which generates local velocity field and density fluctuations [34]. There are also studies showing that the collective motility may be related to the instability in the direction arrangement of rod-shaped bacteria. Moreover, the transmission of chemical information, resistance to antibiotics and individual cognitive decision behavior may also be important factors.

\section{EXPERIMENTAL TECHNEQUES}

\section{Experimental systems}

As a model system to study the dynamic biological assembly of active matters, bacteria have several advantages. On one hand, they are relatively easy to culture in the laboratory environment. On the other hand, we can conveniently gain access to gene sequences and biochemistry data of many strains such as Escherichia coli (E. coli), Bacillus subtilis (B. subtilis) and Pseudomonas aeruginosa ( $P$. aeruginosa) to learn their biochemistry characteristics. Accordingly, many techniques that manipulate bacterial individuals or populations to move in the liquid as well as on the solid surfaces [26] have been developed during the past years.

One method for observing active bacterial systems is to disperse a certain concentration of bacterial suspension in 

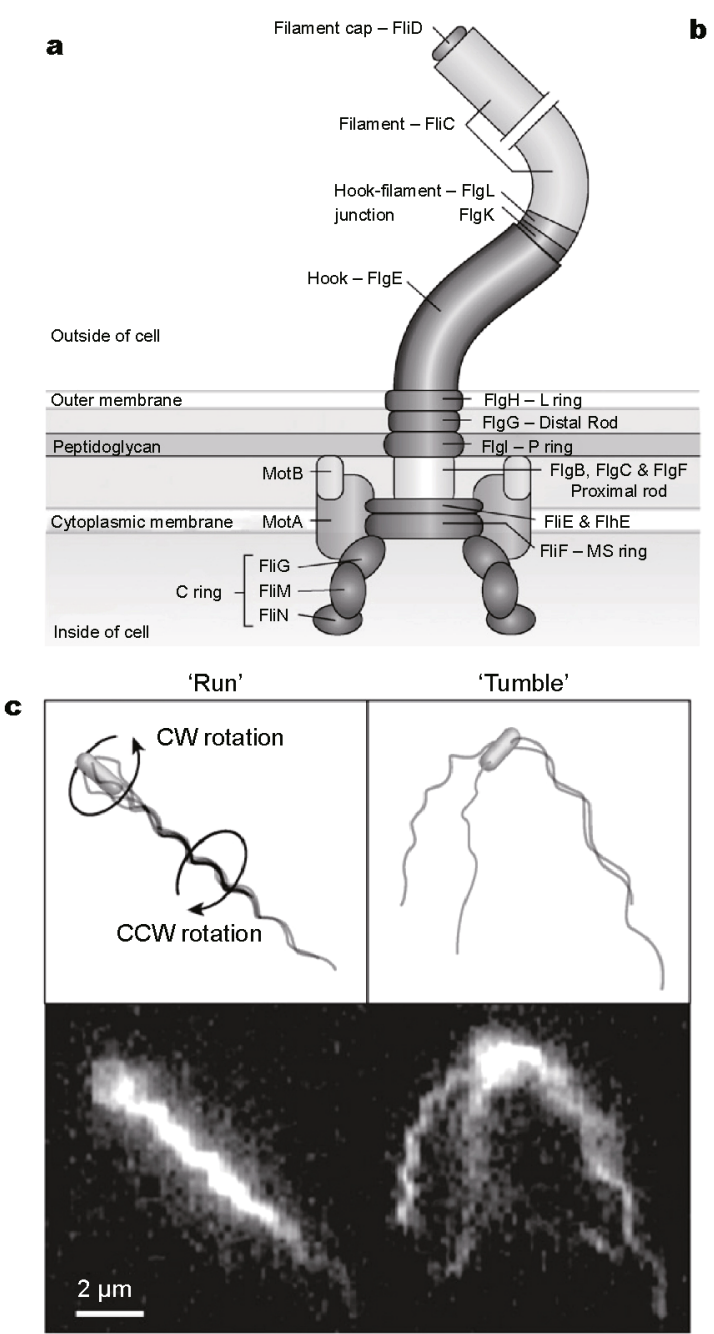

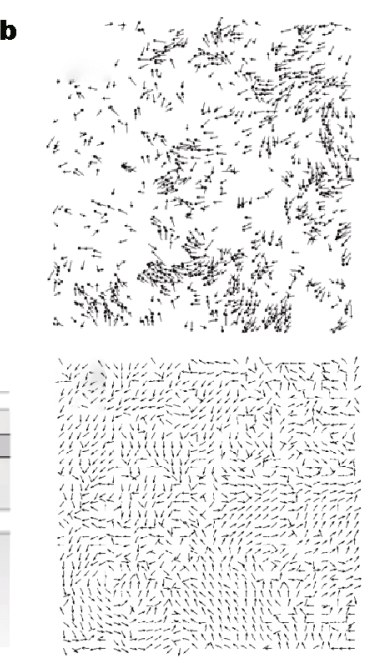

d
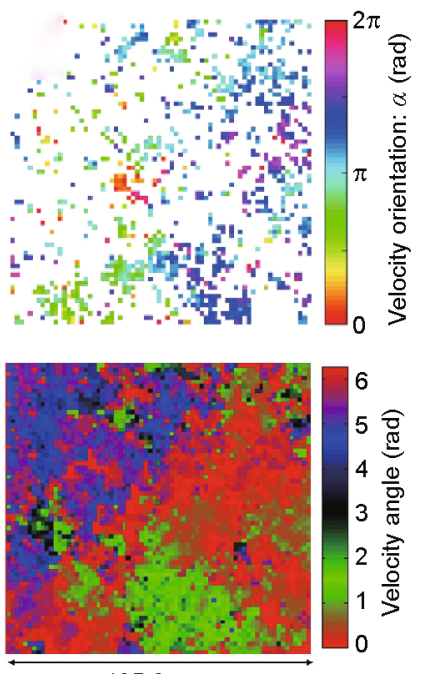

$135.2 \mu \mathrm{m}$

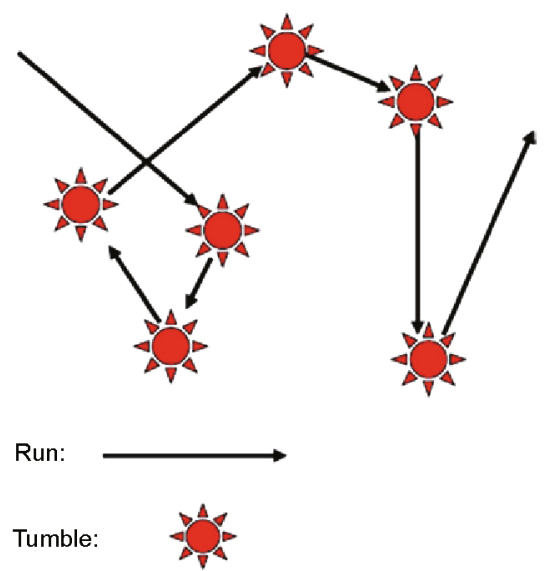

Figure 3 The phenomenon and biological mechanism of collective motility. (a) Structure of bacterial flagellum. (b) Velocity field obtained from PIV and its corresponding color map shown its collective movement. Top panels: $c$ (concentrations) $=1 \%$. bottom panels: $c=2 \%$. (c, d) Fluorescence microscopy images of swimming E. coli cells and "run and tumble mechanism" of single bacterium. (a and c) Reprinted with permission from Ref. [25]. Copyright 2009, the American Institute of Physics. (b) Reprinted with permission from Ref. [32]. Copyright 2014, the Institute of Physics Publishing. (d) Reprinted with permission from Ref. [33]. Copyright 2015, Elsevier.

a langmuir-blodgett (LB) system in a free-standing soap film [35] with thickness about 15-400 $\mu \mathrm{m}$ (Fig. 4). The soap film surface must be free from pressure to maximize the effect of surface-generated velocity gradient. When tracking with micron-sized particles, it is observed that the majority of the bacterial suspension fluid self-organize into an active bacterial film [36]. Another technique is to inoculate bacteria colony on agar surfaces [37], on which the bacteria are generally considered to move on the surfaces instead of entering into the interior.

In the experiments, wild type (WT) B. subtilis and E. coli are commonly used bacterial species. Mutated species could also be used if there are special needs. In general, the thickness of bacteria suspensions is in the level of 3 to 5 microns. For instance, in a commonly used model system, B. subtilis 3610 (WT), the single bacterium is about $0.8 \times 5$ $\mu \mathrm{m}$ in size. They are usually grown on an agar plate which consists of $1 \mathrm{~g} \mathrm{~mL}^{-1}$ peptone and $0.5 \%$ agar, and the temperature is at $30^{\circ} \mathrm{C}$. The thickness of overall fluids is about $3-4$ $\mu \mathrm{m}$. After culturing for about 4 hours, the colony begins to expand outward. Then it can be fetched to observe under a microscope. The behavior of bacterial swarming could be observed at the edge of bacterial colonies.

To observe the collective movement of bacteria, proper control of certain important experimental conditions will be beneficial to the observation of the swarming phenomenon: (1) the nutrient around the bacteria colony can not be too abundant to inhibit their reproduction, otherwise the 


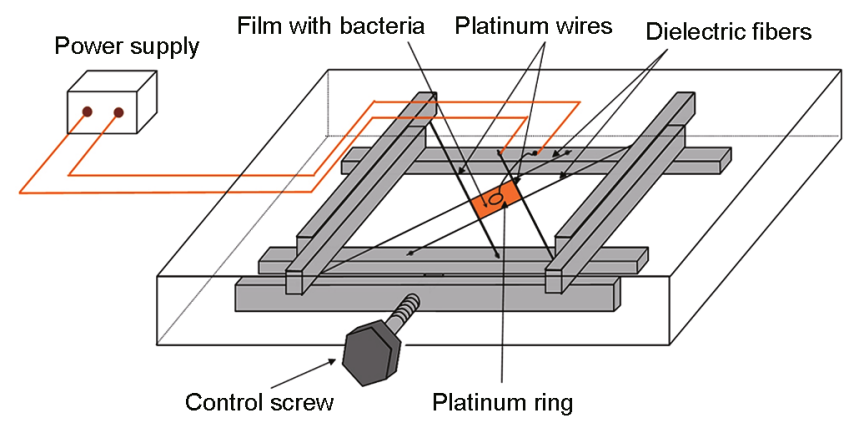

Figure 4 Free-standing bacterial film fabrication device. Reprinted with permission from Ref. [35]. Copyright 2012, Elsevier.

bacteria group will grow so fast and become too concentrated to observe; (2) appropriate temperature and humidity are needed, however, if there is no special heating device, one should control the observation time within a certain period to avoid the decreasing of swimming activity; (3) when high intensity laser illumination is utilized, phototoxic of bacteria should be taken into account; (4) to prevent oxygen from exhausting, the observation time should be controlled properly to maintain bacteria activity; (5) finally, the type and the concentration of agar used should be paid attention since the hardness of solidified agar surface could potentially influence the swarming behavior.

In order to monitor the behavior of individual bacteria, fluorescent labeling method is often used. For example, in one study, red fluorescent proteins were tagged on to ppsB::PtrpE-mCherry gene expressed proteins and the WT was mixed with labeled bacteria at a ratio of 100:1 [38]. Moreover, fluorescence labeling could be performed in different parts on the body such as the head, body or tail as long as it does not affect the motility of bacteria (Fig. 5).

\section{Imaging technologies}

The most commonly used observation technique is phase contrast microscopy. Fluorescence microscopy and bright field microscopy are also utilized. In any case, high numerical aperture and long working distance objective lenses are necessary to acquire high quality images. The fluorescence microscope is mainly used to study the movement of labeled individual bacteria in the population. By this way, the relationship between the velocity direction of bacteria and the direction of surrounding flow field could be analyzed. Compared to fluorescent microscope, the phase contrast microscope could hardly distinguish the individual from large populations. However, it is of great advantage to observe the overall motion. There is one point which needs attention that if the observed range is too small, it may cause the observed phenomenon too localized. To solve this
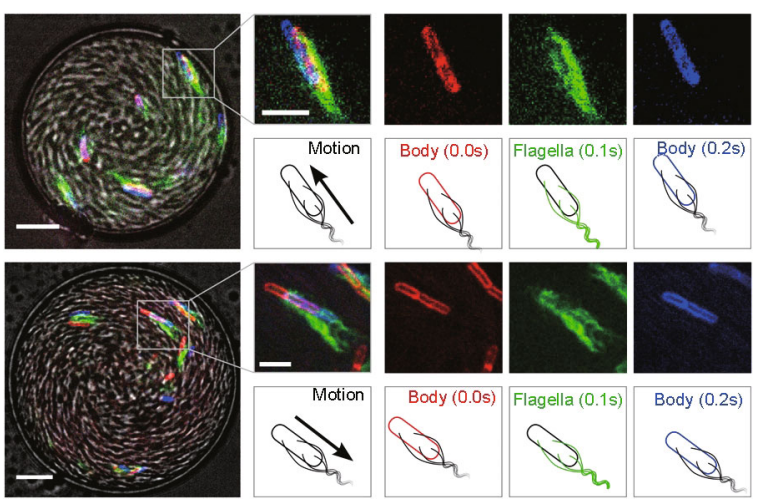

Figure 5 Fluorescently labeled bacteria imaging and its movements. Reprinted with permission from Ref. [39]. Copyright 2014, the American Chemical Society.

problem, sometimes one needs to scan the observation window to different regions and finally stich the captured images together.

\section{DYNAMIC ASSEMBLY BEHAVIOR OF BACTERIA}

\section{The universal phenomenon of collective motion}

Researchers have studied the concentration dependence and the effect of oxygen consumption on the overall movement of bacteria to reveal physical properties of the system. For example, in 2007, Sokolov et al. [40] built a novel thin-film device that could utilize the electric field to control the $\mathrm{pH}$ gradient in the film and herd $B$. Subtilis bacteria into condensed populations of adjustable concentration. They found that the increase of the bacterial concentration did not cause a sharp transition of the correlation length (spatial scale of the collective motion), but their movement became more monotonous and gentle. Later on, in 2012, Sokolov et al. [35] continued to study the B. Subtilis system and found that the consumption of oxygen would affect the swimming speed of bacteria as well as the structure of flow field (Fig. 6a-f). By calculating the spatial correlation length and the temporal autocorrelation function, it was found that the correlation coefficients in time and space are not related to each other beyond the velocity and concentration thresholds. It is concluded that the correlation length is only related to the shape and size of swimming microorganisms themselves.

When bacteria are cultured on agar, varying degrees of coordinated movement of bacteria have been observed by a number of researchers. Therefore, there has been a lot of study on the characteristics of the apparent collective state 

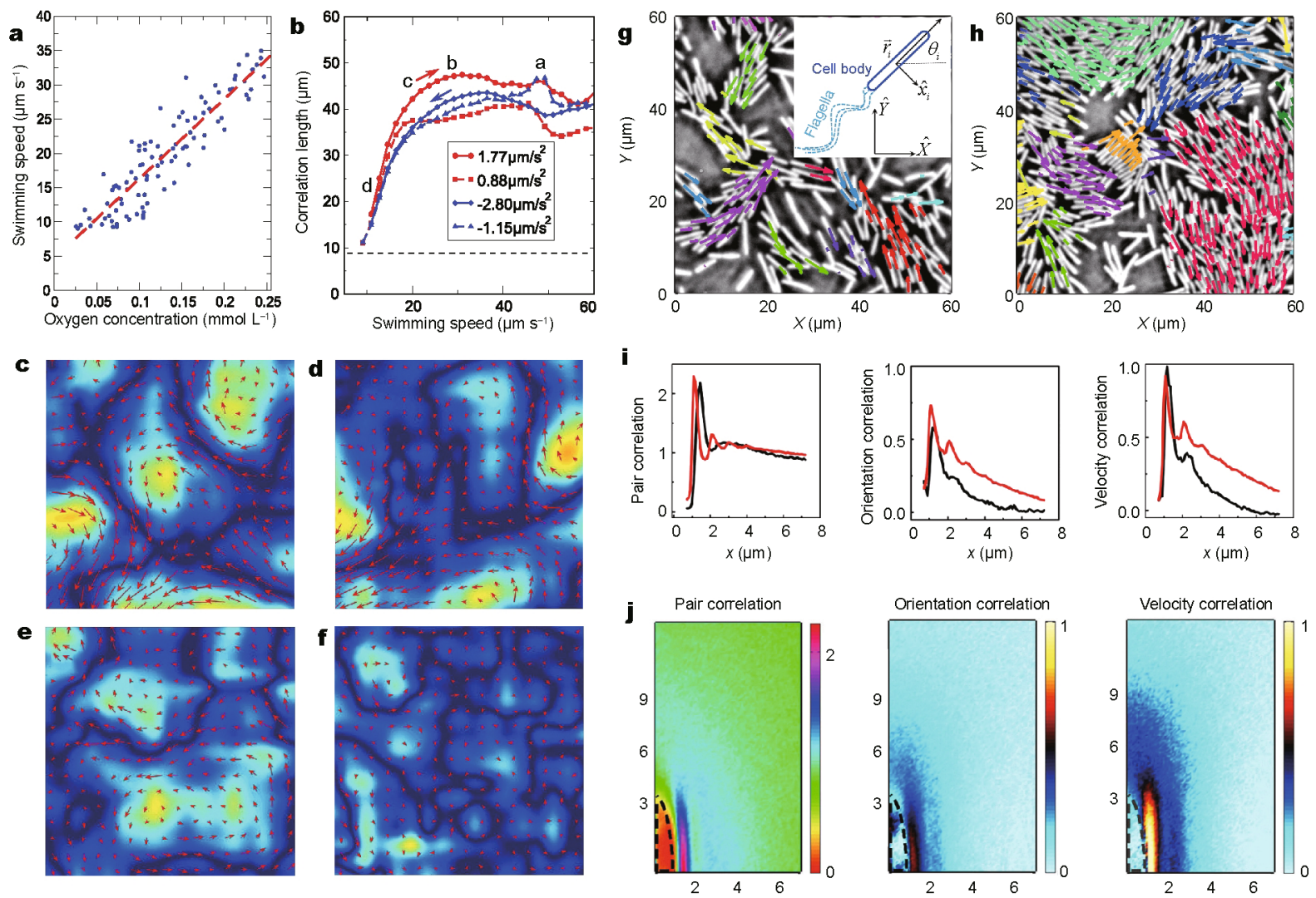

Figure 6 The universal phenomenon of collective motion and its influencing factors. (a-f) The effects of oxygen concentration. (a, b) The relationship between $\mathrm{O}_{2}$ concentration, swimming speed and correlation length. (c-f) The corresponding flow field structures in speeds that are indicated in b. (g, h) B. subtilis colony of two different densities [41]. (i, g) Two-dimensional pair orientation and velocity correlation. (a-f) Reprinted with permission from Ref. [35]. Copyright 2012, the American Physical Society. (g-j) Reprinted with permission from Ref. [8]. Copyright 2010, the American Chemical Society.

and swarming behavior. Zhang et al. [8] used a MAT$\mathrm{LAB}$ algorithm to process images to distinguish bacterial individuals in condensed populations. They reported simultaneous measurements of their location, speed, and direction correlation as a function of time for thousands of WT B. subtilis bacteria within a colony. The bacteria spontaneously assembled into closely packed dynamic clusters and have "giant number density fluctuations" when they moved cooperatively (Fig. 6h-k).

Chen et al. [41] found that with increasing bacterial concentration the velocity of $E$. coli within the plane exhibited a weak synchronization behavior, which lasted at least half an hour. It is interesting that the probability of quasi clockwise movement in different colonies is almost equal. By tracing with silicone oil drops in surface layer, it was found that they moved along an elliptical curve and had the same phase although hundred microns apart. The drop motion with a certain period, amplitude and phase was fitted with a sinusoidal curve, and it was surprised to find that the movement of the bacteria was completely coincident with the movement of the silicone oil drops. Furthermore, while observing in a larger size of area, they found that the collective oscillation evolved into a centimeter-scale travelling wave at different locations and with different periods.

The role of bacterial individuals among populations in collective motility

The occurrence of bacterial swarming behavior is closely related to the movement pattern of bacteria. Fluorescence labeling of bacteria can be used to study the association and contribution of bacterial individuals' behavior to overall generated flow in swarming (Fig. 7a-c). Ariel et al. [42] found that individual E. coli bacteria were performing super-diffusion that was called "levy walks" (LW). While the individuals move at a fixed speed during a longer duration and change the direction randomly, they behave consistently with the so-called LW behavior". Their distribution fits in the "power-law distribution" (Fig. 7d). The authors concluded that this behavior may be related to the foraging of bacteria or the removal of harmful substances. In 2016, 
$\mathbf{a}$
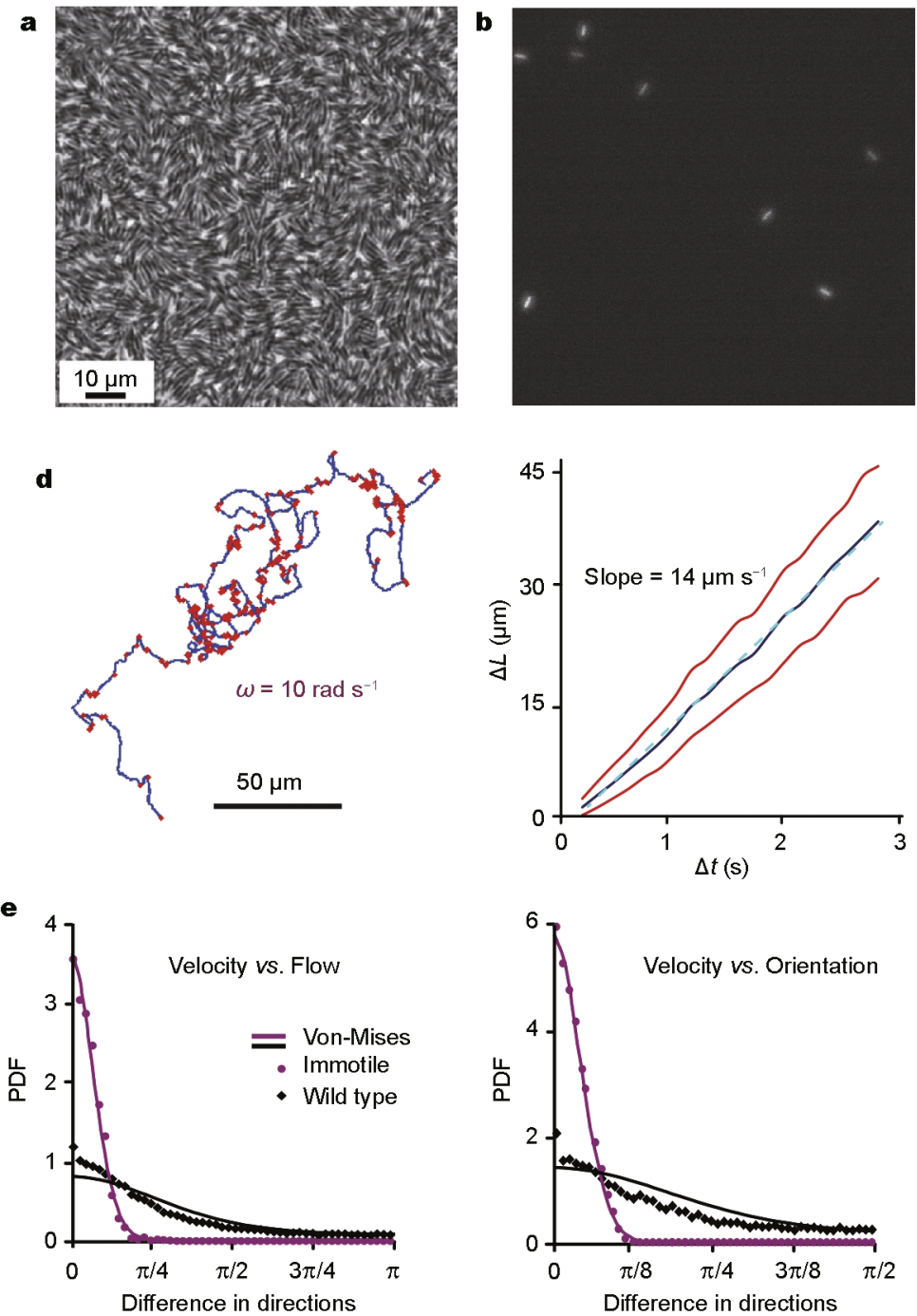
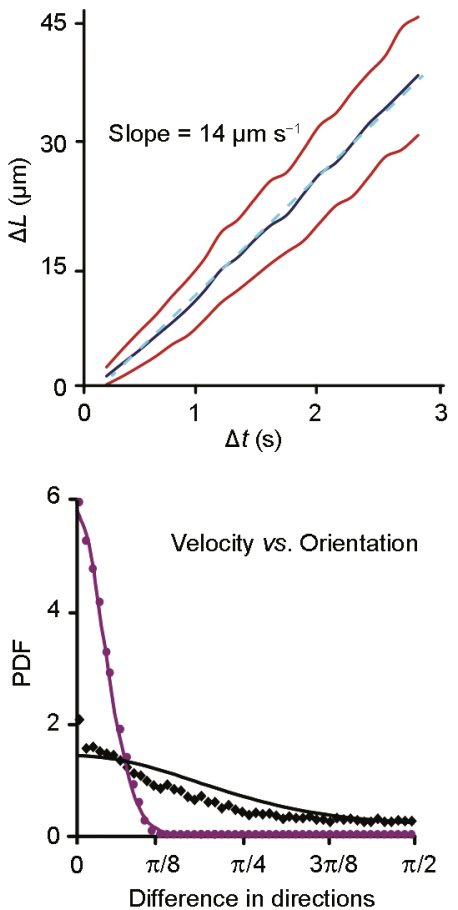
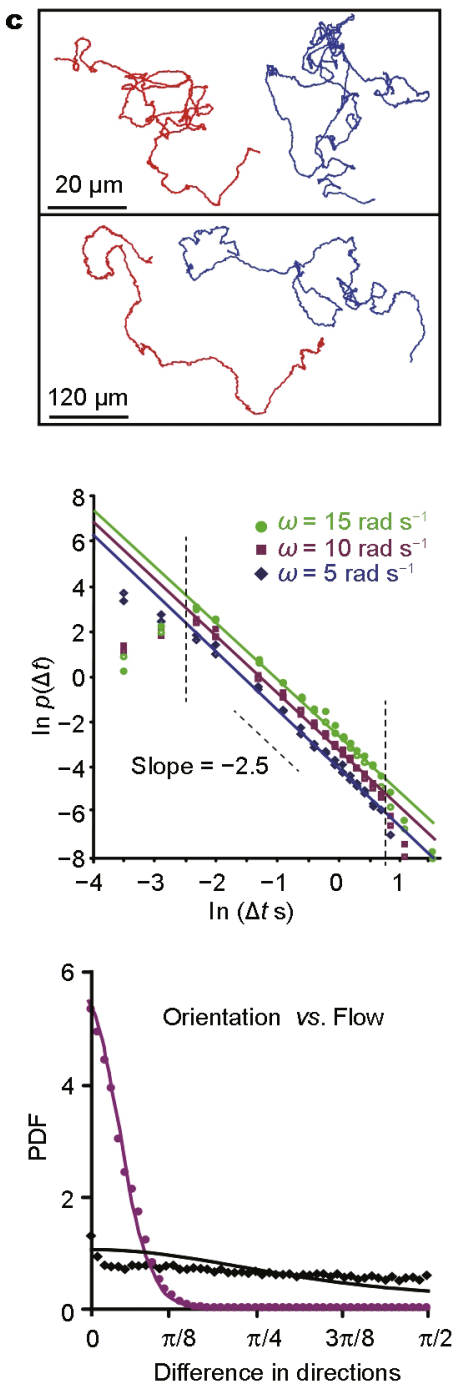

Figure 7 The role of bacterial individuals among populations in collective motion. (a) Phase contrast imaging of a WT B. subtilis swarming colony. (b, c) Fluorescently labeled bacteria and its trajectories. (d) Bacterial trajectories shown "LW characteristics". (e) Comparison of movements between immotile and motile bacteria. (a, b, e) Reprinted with permission from Ref. [38]. Copyright 2016, Elsevier. (c, d) Reprinted with permission from Ref. [42]. Copyright 2015, Nature Publishing Group.

Ryan et al. [38] mixed motile B. subtilis strain 3610 (WT) and the immotile bacteria Serratia marcescens strain 274 (WT) in order to investigate the dynamics of a single bacterium in the populations. It was shown that the angular distribution between the bacterial rotation rate, the direction of the bacteria, and the direction of the surrounding flow field were all Gaussian-like for immotile bacteria. On the contrary, for the motile bacteria, they exhibited abnormal non-Gaussian deviation along with generated flow perpendicular to the collective flow field (Fig. 7e). Therefore, it is possible that the bacteria in the local flow field can control their own direction and may have their own decision.

\section{Characterization of bacterial active fluids using passive tracking particles}

In the study of active fluids like active bacterial fluids, in order to study their non-equilibrium and asymmetry breaking properties, micron-sized particles like polystyrene spheres are often mixed in active fluids as passive tracers [43]. It is found that in the C. reinhardtii system $[29,44]$, the distribution of the displacement of particles often showed a non-Gaussian tail during a short time. For longer time scale, the diffusion constant $D_{\text {eff }}$ of particles is often larger than the diffusion constant $D_{0}$ of Brownian motion. These phenomena are just the characteristic of the active fluids under non-equilibrium state. Many 
people have studied influences of bacterial concentrations on particle diffusion in this field. For example, in the suspensions of $E$. coli, $D_{\text {eff }}$ was found to increase linearly with increasing bacterial concentration $[45,46]$. It was later found that this result was obtained at low concentrations and in the absence of cluster movement. Also, researchers experimentally investigated the effect of particle size on their motion in the suspensions of bacteria. When 0.6 to $39 \mu \mathrm{m}$ particles were mixed with E. coli bacteria [45], it was found that large particles moved faster, which were inconsistent with the principle that the speed of particle is inversely proportional to the radius of the particle according to the traditional Einstein-stokes Equation (Fig. 8). Because the collective movement of active bacterial fluids will clearly affect the degree of rotational freedom of the system, exploration of the rotational diffusion coefficient of anisotropic particles in the active system is meaningful. For instance, Peng et al. [36] have shown that anisotropic ellipsoidal polystyrene (PS) microspheres incorporated into the bacterial film exhibited enhanced translational and rotational diffusion behavior.

The active system of self-driven particles is usually divided into two types depending on whether the generated flow around them are stretched or pulled, i.e., "pusher" or "puller". It was found that the translation and rotation coefficient of the microspheres were negatively correlated in the active system of the pusher type system such as E. coli. In contrast, there is a positive correlation in the puller type bacterial system such as C. reinhardtii [36].

Bacterial antibiotic resistance and collective movements It is well known that the bacterial systems can produce group motility under the condition of antibiotic induction
[48] based on the "run and tumble" mechanism [33]. In sparse bacterial suspensions, the deviation of bacterial voyage was caused by chemical signaling. However, with increased bacterial concentration, the chemical signal is no longer directly controlled by chemotaxis, which can be confirmed by their super-diffusive behavior. In a general biological sense, the swarming of bacteria is likely to be a strategy for resisting antibiotics. For example, Butler et al. [30] found that densely packed bacteria and high-speed swarming behavior favored the survival of bacterial populations in high concentrations of antibiotics.

More importantly, it was found that when bacteria were exposed to sub-lethal doses of kanamycin, the velocity field distribution changed from a normal Boltzmann distribution to a non-Boltzmann distribution [47], due to the aggregation of sub-groups with declined moving ability caused by cluster formation. Moreover, in this process, although antibiotics affect bacteria's swimming speeds apparently, the entire bacterial colony's growth and reproduction has not been greatly threatened (Fig. 9).

\section{Collective behavior of bacteria in confined space}

In nature, bacteria live and forage in porous soil and self-organize into biofilm for resistance. These biological behaviors are all associated with their living environments. Similarly, collective movements of bacteria are found closely related to the environment, which includes both chemical deposits in surroundings and geometrical constraints [24]. In recent years, it has been confirmed that the geometrical constraints around the bacteria havs a great influence on their active flow and also collective state. However, the mechanism of hydrodynamic interactions resulting in this phenomenon still remains unclear.

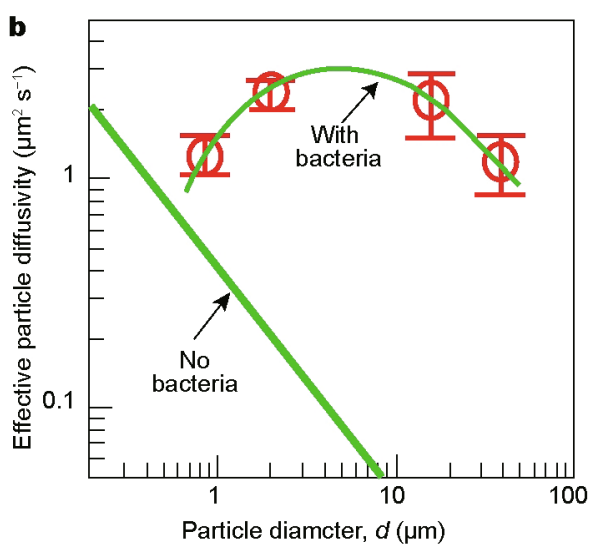

Figure 8 Characterization of bacterial active fluids using passive tracking particles. (a) Captured phase contrast image. (b) Dependence of tracers' diffusivity on particle size. Reprinted with permission from Ref. [45]. Copyright 2016, the Royal Society of Chemistry. 
a

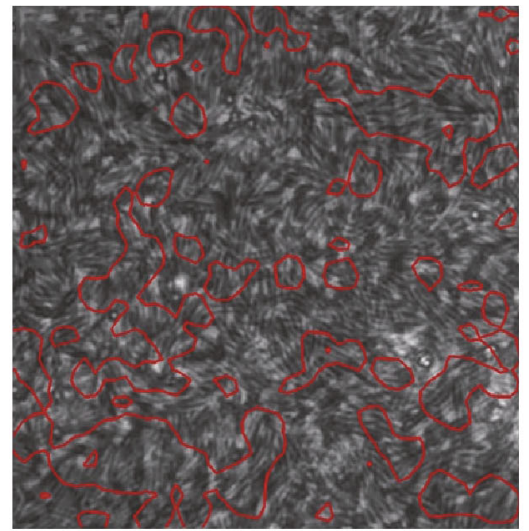

c

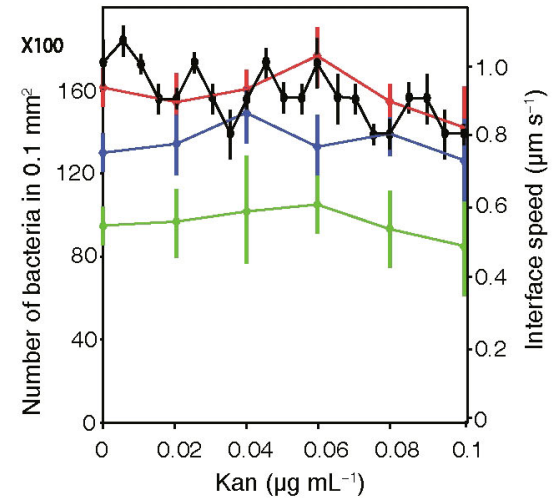

b

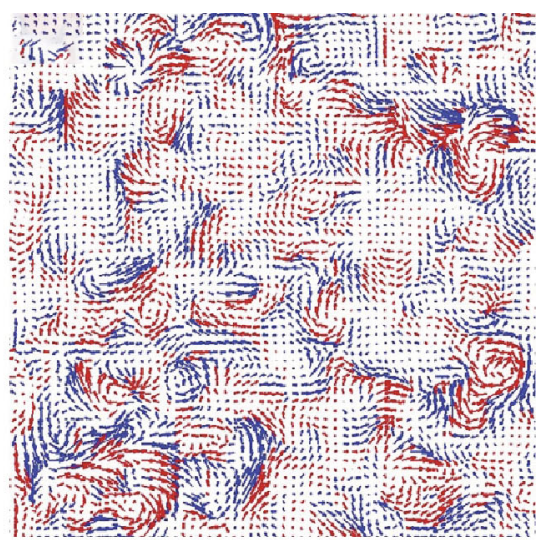

d

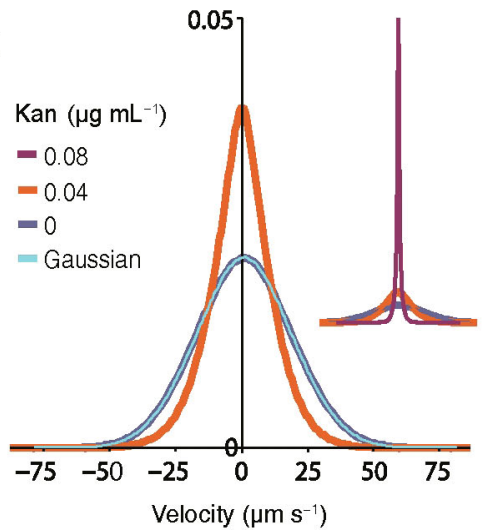

Figure 9 Bacterial antibiotic resistance and collective movements. (a, b) With added $0.08 \mu \mathrm{g} \mathrm{mL}^{-1}$ kanamycin bacteria move in red circled area and its corresponding velocity field. (c) The colony's expansion is not affected by kanamycin. (d) The centered distribution of the $x$ and $y$ components of the velocity. The 0-kanamycin curve is Gaussian. Reprinted with permission from Ref. [47]. Copyright 2015, the American Physical Society.

There are many ways to create a limited space, for example, fabricating simple planar walls [49], circular boundaries, or microfluidic channels [24] with different shapes (Fig. 10a, b). Lushi et al. [39] found that the bacteria self-organized to form "a spiral vortex" when limiting high concentrations of B. subtilis bacteria to a thin cylindrical chamber. They also found that the movement direction in the edge of the population and the center are exactly opposite to each other. On this basis, they constructed a minimum model system based on particle motion, predicting the morphology of cluster motion under the constraints of such circular boundaries very well (Fig. 10c). In the fluorescently labeling experiments, the direction of the body and flagellum are shown to verify the models, revealing interactions among bacterial individuals themselves, as well as hydrodynamic interactions between bacteria and fluid, which are important to the collective state.

Since the bacteria live in the soil pores, fabricating microfluid channels that simulate their living environments have become an important method to study relationships between collective swimming movements and the environ- ment. Wioland et al. [50] found that in a long and narrow circular microfluidic channel, the bacteria could form a stable non-drainage cycle. With the variation of width of the microchannel, the pattern of the movement of bacteria would change from small swirls beginning at the channel width of 35-45 $\mu \mathrm{m}$ to large-scale vortices at 70-90 $\mu \mathrm{m}$. There is no difference with the behavior of the unrestricted system when the width is larger than 90 microns (Fig. 10d). Many studies found that at the edge of the microchannel, the flow field direction produced by bacteria was opposite to the bacterial swimming directions. In another work, Wioland et al. [51] characterized the non-equilibrium state by using thermodynamic equilibrium theory. Based on the Lattice field theories (LFTs), they designed two-dimensional lattice microfluidic channels with a diameter of 50 and $18 \mu \mathrm{m}$ deep, and the lattice was connected with a certain gap. It was found that when the gap width was less than $20 \mu \mathrm{m}$ the self-organization is "vortex". When it was more than $20 \mu \mathrm{m}$, the spontaneous "vortex" disappeared, and instead, the bacteria would flow freely in the lattice. In addition, the author investigated the spin ferromagnetism 

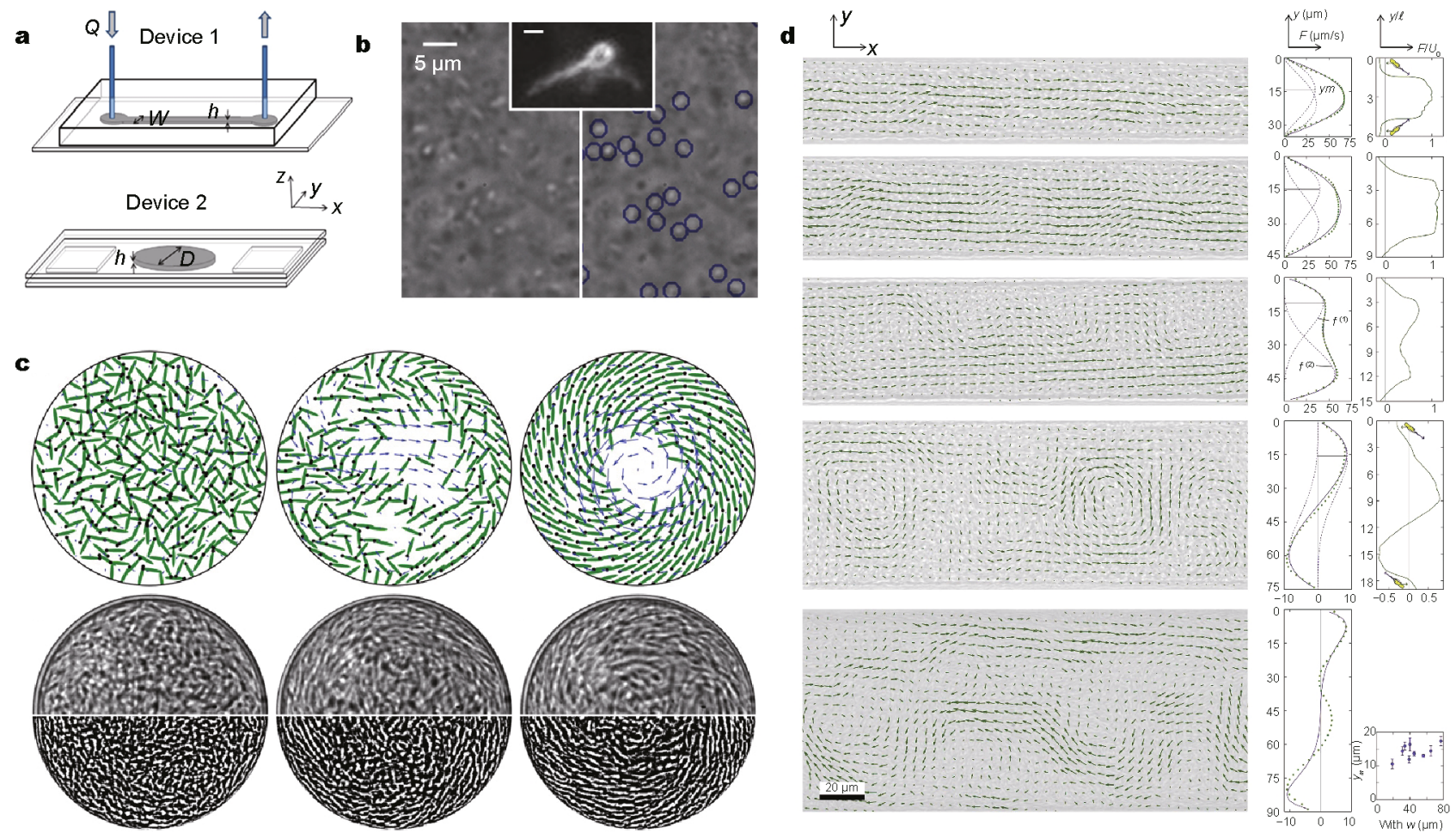

Figure 10 Bacterial collective behavior in confined space. (a) Two simple examples of microfluidic devices [32]. (b) Collective movements in the channel and fluorescently labeled bacteria (inset) [32]. (c) Simulation (top panel) and phase contrast image show the motility with circular boundaries [39]. (d) Universal movement in channels with different widths. (a, b) Reprinted with permission from Ref. [32]. Copyright 2014, the Institute of Physics Publishing. (c) Reprinted with permission from Ref. [39]. Copyright 2014, the American Chemical Society. (d) Reprinted with permission from Ref. [50]. Copyright 2016, the Institute of Physics Publishing.

and they found an interesting phenomenon: different geometric topologies of the lattice influenced bacterial lattice spin direction distribution. This utilization of microfluidic channels to study the dynamic assembly of bacteria could better reveal the relationships between the movement of bacteria, the surrounding flow field, and the entire channel circulation of the network. In particular, some researchers control the aggregation and dispersion of crowded passive particles by adjusting the optical potential of spatial light in active bacterial system. Theoretical studies have shown that the existence of spatial light chaos will greatly affect the movement of active matters. Pince et al. [52] studied the aggregation and dispersion behavior of colloidal particles in hot baths and active bacterial fluids when controllable roughness of the spatial light is introduced by optical potential well. They found that the existence of spatial chaos controlled by external field did change the long-range coupling behavior of the active system. Even more surprisingly, in the active bacterial flow, when the spatial disorder was added to the smooth attractive optical potential with a speckle-like pattern, the change in particles from dispersion to aggregation could be achieved.

\section{DATA ANALYSIS AND PROCESSING}

\section{Phenomenological analysis and statistical description}

To elucidate the collective movements of bacteria, particle imaging velocity (PIV) or optical flow [53] have been utilized as a general method to describe the flow field. This method can clearly illustrate the flow field around the moving bacteria populations with small dense needle-like patterns. Another widely used method to describe bacterial collective oscillation is temporal and spatial correlation [54]. By defining the correlation length and comparing it with the size of the bacteria itself, the spatial scale of collective state is shown. The changes in cluster movement can be effectively measured by calculating bacterial velocity, direction, body orientation correlation function in time and space. Pattern recognition of a single bacterial trajectory can be readily realized by software. It is easy to calculate the distribution of the mean square displacement, translation and rotation of the bacteria movement from the trajectory to obtain the distribution of its motion. When tracking the collective state of bacteria with passive particles, the method of studying the trajectory diffusion 
behavior of the particles is the same as the method of studying the trajectories of individual bacteria. Since the motion of the bacteria has the nature of the vortex, the direction of movement of the tracing particles sometimes can be fitted to a curve of sinusoidal cosine with a certain phase and period.

\section{Physical models and numerical simulation}

In general, the mechanism of active bacterial swarming is considered to consist of two main kinds of interactions: the long-range hydrodynamic interactions and the short-range steric effects or collisions. However, there remain many questions: which dynamic plays a key role? How to construct a physical model to explain the mechanism of coordination movement in active bacteria fluids and to predict the formation of a large number of dynamic vortex? Physicists have done many works to solve the problem.

In low Reynolds number $(R e)$ fluid systems, bacteria are often defined as "dipole" structures [34]. Part of the species like B. subtilis bacteria whose flagella is in the back generates forward thrust to its body. Others such as C. reinhardtii result in pulling force because of its long front flagella. Through this mechanism, bacteria are divided into two categories: pusher and puller. As a consequence, the effect of the surrounding fluid field can also be divided into two types, "extensive" and "contractile" (Fig. 11a).

The swarming behavior of bacteria is usually studied in the field of hydrodynamics. One of the most important features is that it is in a relatively low Reynolds-state fluid environment $\left(\mathrm{Re}=10^{-4}-10^{-2}\right)[55,56]$. The Reynolds number is a parameter that characterizes the fluid properties, physically representing the ratio of the inertial force to the viscous force level. Here, $\operatorname{Re}=\rho v L / \mu$, where $\rho$ and $\mu$ are the fluid density and the dynamic viscosity coefficient, and $v$ and $L$ are the characteristic velocity and characteristic length of the flow field. This characteristic leads to the Stokesian dynamics $[57,58]$, in which the description function of incompressible fluid and the Langevin Equation of Brownian particles are utilized. In addition, the active particles are seen as discrete and the surrounding fluid is treated as an incompressible continuum fluid.

In constructed physical model, single bacterium is directly simplified into self-propelled particles with kinetic equations, for instance, Langevin Equation. This simplification may have different forms such as "point dipole", "slender bodies" [60], "dumbbell” [59] (Fig. 11b), "ellipsoid", "swimming models", and "rigid self-propelled rods". Different physical aspects such as hindering effects and collisions, interactions with fluids (Fig. 11c, d) and the addition of boundary effects due to constraints have got a lot of attention.

Under the premise of the minimal model [61], the continuum model theory $[1,55,59,62]$ utilized to simulate the condition of active bacterial systems has become a mainstream. A two-phase separation model which regards fluid and bacteria as independent interpenetrating continuous a
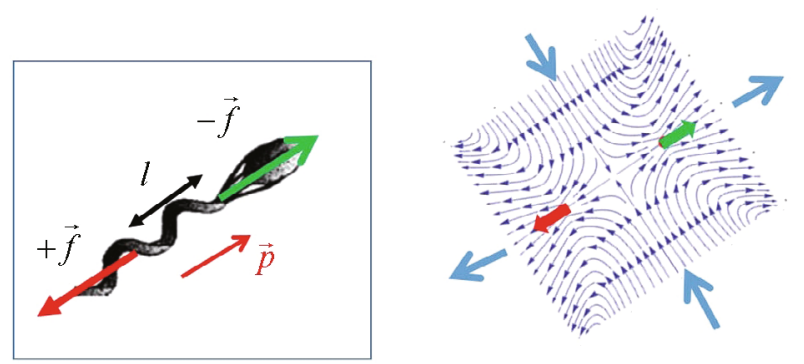

c

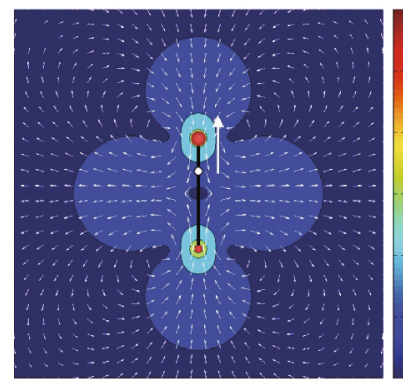

d

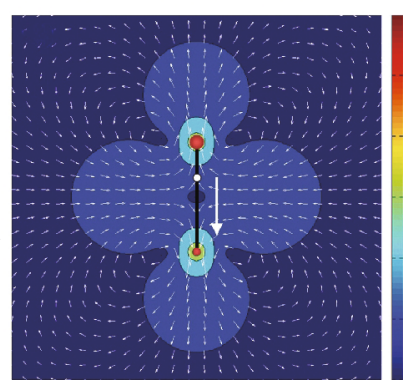

Figure 11 Examples of physical models. (a) Hydrodynamic representation of the bacterium as a "pusher" (left panel) and resulting flow field created by the force dipole imposing on the fluid. (b) Dumbbell models composed of a small and a large sphere of radii $a_{\mathrm{S}}$ and $a_{\mathrm{L}}$, respectively, connected by an infinitely thin rigid rod. (c, d) Flow fields that "a single dumbbell type puller (c) or pusher (d)" exerts on the surrounding fluid. (a) Reprinted with permission from Ref. [34]. Copyright 2016, Springer. (b-d) Reprinted with permission from Ref. [59]. Copyright 2009, the American Chemical Society. 
phases [63] has been proposed, revealing the important effect of the three factors on the vortex pattern: the dipole strength of the bacteria on the fluid, the alignment caused by the entropy effect and the torque due to the fluid gradient. In addition, the mean field theory [62] is often used to describe the stochastic behavior of single or pairwise bacterial motions. Dunkel et al. [64] constructed a minimal fourth-order vector-field theory for incompressible bacterial dynamics, which described the quantitative data of the experiments very well.

\section{OUTLOOKS}

Bacterial swarming behavior is inseparable with local environment such as long-range hydrodynamic interactions, short-term physical interactions and the role of interfaces. Though the research in this field has mostly focused on the universal coordination of bacterial motility, it can still be seen that the coordination and oscillations of the bacterial movement pattern among whole populations has giant difference with the motility of the individuals and disobeys the general physical laws of common dynamic process surprisingly. There still remain a number of fundamental problems to be solved. On one hand, nowadays the utilization of existing physical modeling methods has been limited by many factors in further revealing the regulation laws as well as dynamic relationships between individuals and cluster movements. Therefore, hard efforts should be exerted on theoretical innovations in physical modeling as well as numerical simulations in order to improve their explanation and prediction ability for experiments. On the other hand, currently it is still rare to use external field to control system in time and space in order to observe the dynamic changes in the experimental system.

Overall, a general rule underlies all these uncommon phenomena should be brought to light. In recent years, big data methods such as data-based machine learning have become popular in the field of data mining and analysis. In the future, it is expected that the algorithm of machine learning can be used in the pattern recognition of bacteria swarming and passive particles tracking. For example, some researchers have developed decision trees and random forest algorithms to handle such tasks [65].

Besides the behaviors of individual bacteria themselves, it is crucial to understand the properties of their surrounding fluidic medium. So far people have been using micron-sized passive particles to trace the hydrodynamics of the bacterial environment. During the past decade, our research group have developed a series of nano-imaging techniques capable of tracing single plasmonic nanoparticles with high sensitivity and high resolution [66-71]. Inspired by single particle tracing at the micron-scale, it is expected that a lot of new information on the collective motion of bacteria could be obtained by tracking the spatial and temporal variation of multiple single nanoparticle tracers at the nanoscale. Exploring influences of spatial or boundary confinement as well as external fields on the bacterial active fluids and the diffusion of nanoparticles are also very helpful to go a step further in this field. Besides fundamental insights into the complexity of life, these deepened understandings would help people to build novel active micro/nano motors and complex devices by mimicking the essential functions of their natural counterparts for biomaterial and biomedical applications [72]. We should have reason to believe that advancement in experimental and data analysis techniques will bring us new discoveries in this vast virgin land.

Received 18 April 2017; accepted 31 May 2017; published online 28 June 2017

1 Dombrowski C, Cisneros L, Chatkaew S, et al. Self-concentration and large-scale coherence in bacterial dynamics. Phys Rev Lett, 2004, 93: 098103

2 Ben-Jacob E, Levine H. The artistry of nature. Nature, 2001, 409: 985-986

3 Patteson AE, Gopinath A, Arratia PE. Active colloids in complex fluids. Curr Opin Colloid Interface Sci, 2016, 21: 86-96

4 DeCamp SJ, Redner GS, Baskaran A, et al. Orientational order of motile defects in active nematics. Nat Mater, 2015, 14: 1110-1115

5 Sanchez T, Welch D, Nicastro D, et al. Cilia-like beating of active microtubule bundles. Science, 2011, 333: 456-459

6 Drescher K, Shen Y, Bassler BL, et al. Biofilm streamers cause catastrophic disruption of flow with consequences for environmental and medical systems. Proc Natl Acad Sci USA, 2013, 110: $4345-4350$

7 Chatterjee R, Joshi AA, Perlekar P. Front structure and dynamics in dense colonies of motile bacteria: role of active turbulence. Phys Rev E, 2016, 94: 022406

8 Zhang HP, Be'er A, Florin EL, et al. Collective motion and density fluctuations in bacterial colonies. Proc Natl Acad Sci USA, 2010, 107: 13626-13630

9 Zhou S, Sokolov A, Lavrentovich OD, et al. Living liquid crystals. Proc Natl Acad Sci USA, 2014, 111: 1265-1270

10 Notbohm J, Banerjee S, Utuje KJC, et al. Cellular contraction and polarization drive collective cellular motion. Biophysical J, 2016, 110: $2729-2738$

11 Schuppler M, Keber FC, Kröger M, et al. Boundaries steer the contraction of active gels. Nat Commun, 2016, 7: 13120

12 Be'er A, Harshey RM. Collective motion of surfactant-producing bacteria imparts superdiffusivity to their upper surface. Biophysical J, 2011, 101: 1017-1024

13 Zhang Y, Zhou N, Li N, et al. Giant volume change of active gels under continuous flow. J Am Chem Soc, 2014, 136: 7341-7347

14 Ebbens SJ. Active colloids: Progress and challenges towards realising autonomous applications. Curr Opin Colloid Interface Sci, 2016, 21: 14-23

15 Dey KK, Wong F, Altemose A, et al. Catalytic motors-quo 
vadimus? Curr Opin Colloid Interface Sci, 2016, 21: 4-13

16 Bouffier L, Ravaine V, Sojic N, et al. Electric fields for generating unconventional motion of small objects. Curr Opin Colloid Interface Sci, 2016, 21: 57-64

17 Qiu F, Nelson BJ. Magnetic helical micro- and nanorobots: toward their biomedical applications. Engineering, 2015, 1: 021-026

18 Gao W, Uygun A, Wang J. Hydrogen-bubble-propelled zinc-based microrockets in strongly acidic media. J Am Chem Soc, 2012, 134: 897-900

19 Scharf B. Real-time imaging of fluorescent flagellar filaments of rhizobium lupini H13-3: flagellar rotation and $\mathrm{pH}$-induced polymorphic transitions. J Bacteriology, 2002, 184: 5979-5986

20 Goldstein RE. Green algae as model organisms for biological fluid dynamics. Annu Rev Fluid Mech, 2015, 47: 343-375

21 Wang Y, Hernandez RM, Bartlett DJ, et al. Bipolar electrochemical mechanism for the propulsion of catalytic nanomotors in hydrogen peroxide solutions. Langmuir, 2006, 22: 10451-10456

22 Manjare M, Yang B, Zhao YP. Bubble driven quasioscillatory translational motion of catalytic micromotors. Phys Rev Lett, 2012, 109: 128305

23 Kearns DB. A field guide to bacterial swarming motility. Nat Rev Micro, 2010, 8: 634-644

24 Harshey RM. Bacterial motility on a surface: many ways to a common goal. Annu Rev Microbiol, 2003, 57: 249-273

25 Copeland MF, Weibel DB. Bacterial swarming: a model system for studying dynamic self-assembly. Soft Matter, 2009, 5: 1174-1187

26 Verstraeten N, Braeken K, Debkumari B, et al. Living on a surface: swarming and biofilm formation. Trends Microbiol, 2008, 16: 496-506

27 Koch DL, Subramanian G. Collective hydrodynamics of swimming microorganisms: living fluids. Annu Rev Fluid Mech, 2011, 43: 637-659

28 Burdick J, Laocharoensuk R, Wheat PM, et al. Synthetic nanomotors in microchannel networks: directional microchip motion and controlled manipulation of cargo. J Am Chem Soc, 2008, 130: 8164-8165

29 Saragosti J, Silberzan P, Buguin A. Modeling E. coli tumbles by rotational diffusion. Implications for chemotaxis. PLoS ONE, 2012, 7: e35412

30 Butler MT, Wang Q, Harshey RM. Cell density and mobility protect swarming bacteria against antibiotics. Proc Natl Acad Sci USA, 2010, 107: 3776-3781

31 Elgeti J, Kaupp UB, Gompper G. Response to comment on article: hydrodynamics of sperm cells near surfaces. Biophysical J, 2011, 100: 2321-2324

32 Gachelin J, Rousselet A, Lindner A, et al. Collective motion in an active suspension of Escherichia coli bacteria. New J Phys, 2014, 16: 025003

33 De Magistris G, Marenduzzo D. An introduction to the physics of active matter. Physica A-Stat Mech Appl, 2015, 418: 65-77

34 Clement E, Lindner A, Douarche C, et al. Bacterial suspensions under flow. Eur Phys J Spec Top, 2016, 225: 2389-2406

35 Sokolov A, Aranson IS. Physical properties of collective motion in suspensions of bacteria. Phys Rev Lett, 2012, 109: 248109

36 Peng Y, Lai L, Tai YS, et al. Diffusion of ellipsoids in bacterial suspensions. Phys Rev Lett, 2016, 116: 068303

37 Darnton NC, Turner L, Rojevsky S, et al. Dynamics of bacterial swarming. Biophysical J, 2010, 98: 2082-2090

38 Ryan SD, Ariel G, Be'er A. Anomalous fluctuations in the orientation and velocity of swarming bacteria. Biophysical J, 2016, 111: 247-255

39 Lushi E, Wioland H, Goldstein RE. Fluid flows created by swim- ming bacteria drive self-organization in confined suspensions. Proc Natl Acad Sci USA, 2014, 111: 9733-9738

40 Sokolov A, Aranson IS, Kessler JO, et al. Concentration dependence of the collective dynamics of swimming bacteria. Phys Rev Lett, 2007, 98: 158102

41 Chen C, Liu S, Shi XQ, et al. Weak synchronization and large-scale collective oscillation in dense bacterial suspensions. Nature, 2017, 542: $210-214$

42 Ariel G, Rabani A, Benisty S, et al. Swarming bacteria migrate by Lévy Walk. Nat Commun, 2015, 6: 8396

43 Zhang HP, Be'er A, Smith RS, et al. Swarming dynamics in bacterial colonies. Europhys Lett, 2009, 87: 48011

44 Leptos KC, Guasto JS, Gollub JP, et al. Dynamics of enhanced tracer diffusion in suspensions of swimming eukaryotic microorganisms. Phys Rev Lett, 2009, 103: 198103

45 Patteson AE, Gopinath A, Purohit PK, et al. Particle diffusion in active fluids is non-monotonic in size. Soft Matter, 2016, 12: 2365-2372

46 Vaccari L, Allan DB, Sharifi-Mood N, et al. Films of bacteria at interfaces: three stages of behaviour. Soft Matter, 2015, 11: 6062-6074

47 Benisty S, Ben-Jacob E, Ariel G, et al. Antibiotic-induced anomalous statistics of collective bacterial swarming. Phys Rev Lett, 2015, 114: 018105

48 Stein T. Bacillus subtilis antibiotics: structures, syntheses and specific functions. Mol Microbiol, 2005, 56: 845-857

49 Eisenstecken T, Hu J, Winkler RG. Bacterial swarmer cells in confinement: a mesoscale hydrodynamic simulation study. Soft Matter, 2016, 12: 8316-8326

50 Wioland H, Lushi E, Goldstein RE. Directed collective motion of bacteria under channel confinement. New J Phys, 2016, 18: 075002

51 Wioland H, Woodhouse FG, Dunkel J, et al. Ferromagnetic and antiferromagnetic order in bacterial vortex lattices. Nat Phys, 2016, 12: $341-345$

52 Pinçe E, Velu SKP, Callegari A, et al. Disorder-mediated crowd control in an active matter system. Nat Commun, 2016, 7: 10907

53 Horn BKP, Schunck BG. Determining optical flow. Artif Intelligence, 1981, 17: 185-203

54 Kitsunezaki S. Spatio-temporal patterns of bacteria caused by collective motion. Physica D-Nonlinear Phenomena, 2006, 216: 294-300

55 Gyrya V, Aranson IS, Berlyand LV, et al. A model of hydrodynamic interaction between swimming bacteria. Bull Math Biol, 2010, 72: $148-183$

56 Ishikawa T, Simmonds MP, Pedley TJ. Hydrodynamic interaction of two swimming model micro-organisms. J Fluid Mech, 2006, 568: 119-160

57 Aranson IS, Sokolov A, Kessler JO, et al. Model for dynamical coherence in thin films of self-propelled microorganisms. Phys Rev E, 2007, 75: 040901

58 Ryan SD, Berlyand L, Haines BM, et al. A kinetic model for semidilute bacterial suspensions. Multiscale Model Simul, 2013, 11: 1176-1196

59 Baskaran A, Marchetti MC. Statistical mechanics and hydrodynamics of bacterial suspensions. Proc Natl Acad Sci USA, 2009, 106: $15567-15572$

60 Shelley M, Saintillan D. Instabilities and dynamics in suspensions of self-locomoting rods. Salt Lake City: the 60th Annual Meeting of the Divison of Fluid Dynamics. New York: American Physical Society, 2007, 006

61 Marchetti MC, Fily Y, Henkes S, et al. Minimal model of active colloids highlights the role of mechanical interactions in controlling 
the emergent behavior of active matter. Curr Opin Colloid Interface Sci, 2016, 21: 34-43

62 Simha RA, Ramaswamy S. Statistical hydrodynamics of ordered suspensions of self-propelled particles: waves, giant number fluctuations and instabilities. Physica A-Stat Mech Appl, 2002, 306: 262-269

63 Wolgemuth CW. Collective swimming and the dynamics of bacterial turbulence. Biophysical J, 2008, 95: 1564-1574

64 Dunkel J, Heidenreich S, Drescher K, et al. Fluid dynamics of bacterial turbulence. Phys Rev Lett, 2013, 110: 228102

65 Damos P. Using multivariate cross correlations, Granger causality and graphical models to quantify spatiotemporal synchronization and causality between pest populations. BMC Ecol, 2016, 16: 33

66 Xiong B, Zhou R, Hao J, et al. Highly sensitive sulphide mapping in live cells by kinetic spectral analysis of single Au-Ag core-shell nanoparticles. Nat Commun, 2013, 4: 1708

67 Xiao L, Qiao Y, He Y, et al. Imaging translational and rotational diffusion of single anisotropic nanoparticles with planar illumination microscopy. J Am Chem Soc, 2011, 133: 10638-10645

68 Xiao L, Wei L, Cheng X, et al. Noise-free dual-wavelength difference imaging of plasmonic resonant nanoparticles in living cells.
Anal Chem, 2011, 83: 7340-7347

69 Peng Y, Xiong B, Peng L, et al. Recent advances in optical imaging with anisotropic plasmonic nanoparticles. Anal Chem, 2015, 87: 200-215

70 Hao J, Xiong B, Cheng XD, et al. High-throughput sulfide sensing with colorimetric analysis of single $\mathrm{Au}-\mathrm{Ag}$ core-shell nanoparticles. Anal Chem, 2014, 86: 4663-4667

71 Cheng X, Dai D, Xu D, et al. Subdiffraction-limited plasmonic imaging with anisotropic metal nanoparticles. Anal Chem, 2014, 86: 2303-2307

72 Peyer KE, Zhang L, Nelson BJ. Bio-inspired magnetic swimming microrobots for biomedical applications. Nanoscale, 2013, 5: $1259-1272$

Acknowledgments This work was supported by the National Natural Science Foundation of China (21425519) and Tsinghua University Startup Fund.

Author contributions Feng J and He Y wrote the paper.

Conflict of interest The authors declare that they have no conflict of interest.

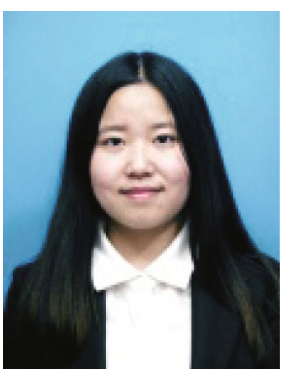

Jingjing Feng received her BSc degree from the Department of Chemistry, Jilin University in 2016. After graduation, she joined the PhD program in Tsinghua University under the supervision of Prof. Yan He. Currently her research interest is focused on probing the dynamic self-assembly of active matters such as self-propelled particles and especially active bacteria systems with single particle imaging techniques.

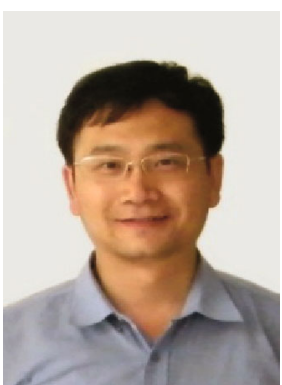

Yan He received his BSc degree in 1995 from Peking University and PhD degree in 2001 from the University of Iowa. During 2002-2005, he worked as a postdoctoral associate at Ames Lab-DOE. After working as a professor in the College of Chemistry and Chemical Engineering at Hunan University during 2005-2015, he joined the Department of Chemistry at Tsinghua University as a professor in 2015. His current research focuses on single molecule imaging and analysis of plasmonic nanoparticles and their applications in biological research.

\section{细菌活性物质的集群行为以及动态组装}

冯靖靖, 何彦*

摘要 近年来由于活性物质体系表现出的复杂动态行为, 对活性物质的研究趋于热点. 细菌活性体系由于鞭毛驱动而产生的在非平衡状态 下的特殊运动行为成为观察复杂体系整体动态组装及集群运动的较好的模型体系, 本综述主要从如下几个方面对细菌活性体系的动态组 装行为进行了简要总结: (1)细菌集群行为的主要现象及生物学机制; (2)研究细菌集群行为的主要技术方法; (3)细菌动态集群行为的相关研 究, 其中包括在相对非限制的空间体系和限制空间体系的研究; (4)在研究动态组装现象时的唯像描述方法以及一些简要的物理模型. 本文 旨在呈现研究细菌动态集群组装行为的研究概貌, 以体现探究过程中所表现出的个体与群体组装行为的动态关联, 希望一窥复杂体系的 整体在动态变化中的规律,包括与周围环境的相互作用等. 